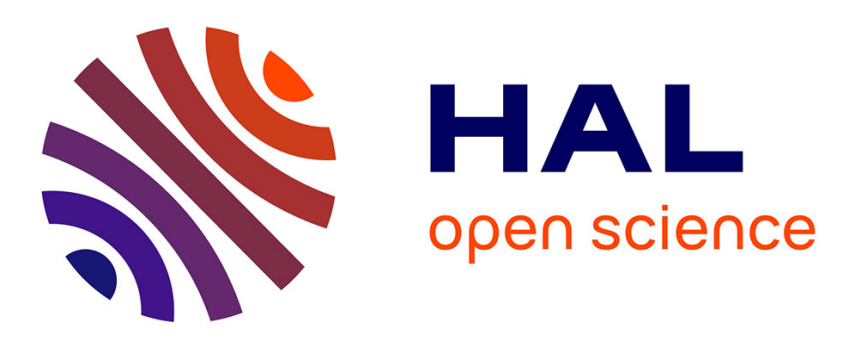

\title{
A Behavioral Assay to Study Effects of Retinoid Pharmacology on Nervous System Development in a Marine Annelid
}

M. Handberg-Thorsager, V. Ulman, P. Tomançak, D. Arendt, Michael Schubert

\section{To cite this version:}

M. Handberg-Thorsager, V. Ulman, P. Tomançak, D. Arendt, Michael Schubert. A Behavioral Assay to Study Effects of Retinoid Pharmacology on Nervous System Development in a Marine Annelid. Methods in Molecular Biology, pp.193-207, 2019. hal-02274987

\section{HAL Id: hal-02274987 \\ https://hal.science/hal-02274987}

Submitted on 29 Oct 2021

HAL is a multi-disciplinary open access archive for the deposit and dissemination of scientific research documents, whether they are published or not. The documents may come from teaching and research institutions in France or abroad, or from public or private research centers.
L'archive ouverte pluridisciplinaire HAL, est destinée au dépôt et à la diffusion de documents scientifiques de niveau recherche, publiés ou non, émanant des établissements d'enseignement et de recherche français ou étrangers, des laboratoires publics ou privés. 


\section{A Behavioral Assay to Study Effects of Retinoid}

\section{Pharmacology on Nervous System Development in A}

\section{Marine Annelid}

M Handberg-Thorsager ${ }^{1, *}$, V Ulman', P Tomançak ${ }^{1}$, D Arendt' ${ }^{2,3}$, M

\section{Schubert ${ }^{4}$}

${ }^{1}$ Max Planck Institute of Molecular Cell Biology and Genetics, Pfotenhauerstrasse 108, 01307

Dresden, Germany.

${ }^{2}$ Developmental Biology Unit, European Molecular Biology Laboratory, Meyerhofstrasse 1, 69012 Heidelberg, Germany.

${ }^{3}$ Centre for Organismal Studies, University of Heidelberg, Im Neuenheimer Feld 230, 69120 Heidelberg, Germany.

${ }^{4}$ Sorbonne Université, CNRS, Laboratoire de Biologie du Développement de Villefranchesur-Mer, Institut de la Mer de Villefranche-sur-Mer, 181 Chemin du Lazaret, 06230

Villefranche-sur-Mer, France.

Running Head: Assay for characterizing behavioral changes of developing aquatic larvae

*Correspondence should be addressed to Mette Handberg-Thorsager.

Address: Max Planck Institute of Molecular Cell Biology and Genetics, Pfotenhauerstrasse 108, 01307 Dresden, Germany.

E-mail: handberg@mpi-cbg.de 


\section{Abstract}

Autonomous animal locomotion, such as swimming, is modulated by neuronal networks acting on cilia or muscles. Understanding how these networks are formed and coordinated is a complex scientific problem, which requires various technical approaches. Among others, behavioral studies of developing animals treated with exogenous substances have proven to be a successful approach for studying the functions of neuronal networks. One such substance crucial for the proper development of the nervous system is the vitamin A-derived molecule retinoic acid (RA) $(1,2)$. In the larva of the marine annelid Platynereis dumerilii, for example, RA is involved in the specification and differentiation of individual neurons and responsible for orchestrating the swimming behavior of the developing larva (3). Here, we report a workflow to analyze the effects of RA on the locomotion of the P. dumerilii larva. We provide a protocol for both the treatment with RA and the recording of larval swimming behavior. Additionally, we present a pipeline for the analysis of the obtained data in terms of swimming speed and movement trajectory. This Chapter thus summarizes the methodology for analyzing the effects of a specific drug treatment on larval swimming behavior. We expect this approach to be readily adaptable to a wide variety of pharmacological compounds and aquatic species.

Key Words 13-cis and all-trans retinoic acid, Behavioral analysis, Live imaging, Marine invertebrate larvae, Movement trajectory, Pharmacological treatments, Platynereis dumerilii, Swimming speed 


\section{Introduction}

Autonomous animal locomotion by swimming is the outcome of a series of interactions between neurons and their projections to and coordination of effector cells, such as ciliary and muscle cells. In the course of development, marine organisms often alternate between ciliaryand muscle-based swimming movements. For example, in the young larva of the annelid Platynereis dumerilii swimming depends solely on the activity of equatorial bands of ciliated cells, which are directly connected to a simple nervous system (4). Active ciliary beating keeps the larva steady in the water column, whereas ciliary arrest leads to the sinking of the larva. Later during development, in advanced larval stages with differentiated muscle cells, movement is accomplished by an alternation of ciliary beating and muscle activity $(5,6)$.

Studies into the molecular mechanisms that coordinate the activity of the nervous system, the ciliary band, and the muscles during larval movement require the development of behavioral assays applicable to a wide array of candidate molecules. Previous studies have shown that larvae of $P$. dumerilii are suitable models for studying the effects of signaling molecules during locomotion. A detailed characterization of the developing neuro-ciliary system, for example, has revealed that the hormone melatonin is crucial for the nocturnal sinking of $P$. dumerilii larvae by increasing the frequency of ciliary arrest (7). Additionally, it has already been established that neuropeptides control the vertical up and down movements of young larvae through the neuro-ciliary system $(\mathbf{8}, \mathbf{9})$. Regarding larval movements mediated by muscles, we have previously shown that the signaling molecule retinoic acid (RA), a derivate of vitamin A and crucially required for orchestrating animal development (1, $2,10)$, is involved in specification and differentiation of the nervous system in older $P$. dumerilii larvae, where locomotion relies both on the neuro-ciliary and neuromuscular systems (3). An increase of RA signaling activity by exogenous treatments with RA thus resulted in morphological malformations of the larval nervous system, which were 
accompanied by a decrease in larval swimming speed and changes in larval swimming patterns (3). The treatment-induced alterations of nervous system topology and connectivity were thus reflected by changes in the swimming behavior of $P$. dumerilii larvae.

Making use of this interconnection between nervous system development and swimming behavior, here, we present a detailed protocol for analyzing the effects of pharmacological compounds on the swimming speed and movement trajectory of aquatic larvae. Based on the example of RA treatments of developing P. dumerilii larvae, we explain how to apply the drug and how to perform live imaging of larval swimming. We further describe a data analysis pipeline for determining the swimming speed and movement trajectory of individual larvae from the movies. To optimize the utility of this protocol, the methodology is based exclusively on commercially available material and open-source software.

\section{Materials}

RA isomers, such as all-trans, 13-cis, and 9-cis RA (respectively referred to here as ATRA, 13cRA, and 9cRA) (11), are light sensitive compounds and, when working with these drugs, care should thus be taken to avoid exposure to light. Reconstitution and aliquoting of stock solutions and dilution to work concentrations are best performed in a dark room under red light. In addition, RA solutions need to be stored in amber, light-tight Eppendorf tubes at $20^{\circ} \mathrm{C}$ or $-80^{\circ} \mathrm{C}$ to optimize drug preservation. Note that the use of freshly prepared and rapidly frozen RA dilutions is highly recommended, as drug activity tends to decrease by repeated freeze-thaw cycles (see Note 1).

RA isomer choice as well as final treatment concentrations vary between organisms $(3,12,13)$ and thus need to be assessed individually for each species (see Note 2$)$. Treatments of $P$. dumerilii embryos and larvae with ATRA and 13cRA, for example, were based on the 
observation that ATRA and 13cRA, but not 9cRA, are present at measurable concentrations in tissues of both developing and adult annelids (3).

As RA is involved in various biological processes, special caution needs to be taken when handling the drug. Wear gloves and work under a fume hood. Residues should be discarded following validated safety protocols.

\subsection{Drug Preparation}

1. Dissolve ATRA (Sigma-Aldrich) and 13cRA (Sigma-Aldrich) in dimethyl sulfoxide (DMSO) or ethanol at a final concentration of $10 \mathrm{mM}$ (stock solution) by vortexing (see Note 3). Aliquot the stock solution into amber Eppendorf tubes (Sigma-Aldrich) and store at $-20^{\circ} \mathrm{C}$ or $-80^{\circ} \mathrm{C}$ until use. Thaw a new aliquot for each experiment and discard the leftover.

\subsection{Incubation Solution}

1. Filter, through a $0.22 \mu \mathrm{m}$ mesh (Corning), the solution to be used for incubation of embryos and larvae during the drug treatment. For marine organisms, either natural seawater (NSW) or artificial seawater (ASW) is used. The water temperature needs to match the culturing conditions (see Note 4).

\section{Methods}

RA treatment experiments are carried out in $6 \mathrm{~mL} \mathrm{NSW}$ in 6-well plates in three biological replicates. For longer drug treatments, keep the number of embryos to 100 to 200 per well. High embryo density negatively affects well-being and development of embryos and larvae. Perform treatment experiments at the culturing temperature of embryos and larvae (see Note 4). 


\subsection{Drug Treatment}

1. Prepare a 6-well plate with $2.5 \mathrm{~mL}$, per well, of filtered $(0.22 \mu \mathrm{m}) \mathrm{NSW}$. Add embryos in $0.5 \mathrm{ml} \mathrm{NSW}$ to the wells (see Note 5) to reach a $3 \mathrm{~mL}$ volume per well and keep them in the culturing incubator. An additional $3 \mathrm{~mL}$ will be added to each well to establish the different treatment conditions (see Subheading 3.1.2). Use two of the wells for ATRA treatment, two for 13cRA treatment, one for a control group treated with DMSO (or ethanol if used for dissolving the retinoids) to control for potential effects of DMSO (or ethanol) on animal development, and one for wild-type embryos to confirm normal development of the embryo batch used for the experiment.

2. Prepare ATRA and 13cRA at final work concentrations of $0.5 \mu \mathrm{M}$ and $1.0 \mu \mathrm{M}$ right before use (see Note 2$)$. Add $3 \mathrm{~mL}$ of filtered $(0.22 \mu \mathrm{m}) \mathrm{NSW}$ to $15 \mathrm{~mL}$ Falcon tubes, preparing one $15 \mathrm{ml}$ Falcon tube per treatment. Subsequently, add $0.3 \mu \mathrm{L}$ ATRA stock solution (at $10 \mathrm{mM}$ ) or $0.3 \mu \mathrm{L} \mathrm{13cRA}$ stock solution (at $10 \mathrm{mM}$ ) to prepare the $0.5 \mu \mathrm{M}$ final work concentrations, $0.6 \mu \mathrm{L}$ ATRA stock solution (at $10 \mathrm{mM}$ ) or $0.6 \mu \mathrm{L} 13 \mathrm{cRA}$ stock solution (at $10 \mathrm{mM}$ ) to prepare the $1.0 \mu \mathrm{M}$ final work concentrations or $0.6 \mu \mathrm{L}$ DMSO (or ethanol) to prepare the final work concentration for the treatment control. Vortex the Falcon tubes immediately to dissolve the drug and transfer, with a Pasteur pipette, the solution in each Falcon tube to the corresponding well of the 6-well plate. This step thus yields a final treatment volume of $6 \mathrm{~mL}$ per well. Gently mix embryos and drug solution with the Pasteur pipette. Make sure to use a clean Pasteur pipette for each experimental condition (i.e. for each Falcon tube).

3. Cover the 6-well plate with aluminum foil to avoid light exposure and incubate at the appropriate culturing temperature. Our RA drug treatments were performed for 24 hours on developing $P$. dumerilii at 48 hours-post-fertilization (hpf). 


\subsection{Live Recording of Larval Swimming}

Use a stereomicroscope with a fast-speed camera (25 frames per second), e.g. a Nikon Digital Sight DS-Fi1 or a DMK 42BUC03 (The Imaging Source). This setup allows the recording of the swimming behavior of the treated larvae (with a size of about $200 \mu \mathrm{m}$ ) both in a horizontal plane (covering a swimming area of $11.34 \mathrm{~cm}^{2}$ ) and, albeit to a lesser extent, in a vertical plane (covering $0.53 \mathrm{~cm}$ of the water column). For more detailed measurements of vertical swimming behavior in the water column, home-built vertical columns need to be used, as described, for example, by Conzelmann et al. (8).

1. Remove the aluminum foil and record the swimming behavior of the larvae in the 6well plate. Record one well at a time and change between plates of the biological replicates for each recording to ensure the same recording conditions (i.e. incubation in dark before recording). Record for one minute at maximum speed. Cover the plate with aluminum foil after recording.

2. Save the files in a file format that can be opened by the program Fiji (14), such as TIFF or JPEG. Make sure to save the metadata to extract the exact time frames, especially if these are not constant. This is important for subsequent speed calculations (see Subheading 3.3.12-3.3.14).

\subsection{Analysis of Larval Swimming Behavior}

Analysis of the swimming behavior can be divided into two parts: measuring the swimming speed and assessing the swimming trajectory. In both cases, tracking of embryos and larvae over time is needed. Different open-source tracking programs are available under Fiji (14), including TrackMate (15) and MTrackJ (16). As TrackMate (15) offers automated segmentation and tracking of individual specimens, it will be used here (see Note 6). 
1. Download and install the required software. Fiji (14) can be downloaded from https://fiji.sc and TrackMate (15) from https://imagej.net/TrackMate. Download also the additional "Track Analysis" plugins under "5.1 Downloadable Jars" at http://imagej.net/TrackMate\#Downloadable jars to be able to extract the "Total Distance Travelled" parameter (see Subheading 3.3.18).

2. Open the previously recorded movie in Fiji (14) and select an area that completely excludes the margins of the 6-well plate (see Note 7). Save the resulting file as TIFF.

3. Launch TrackMate (15). All the operations are performed from the TrackMate window, which appears next to the movie (see Fig. 1). Start by adjusting the image and movie properties, if needed ("Calibration Settings"), and select the region and time points of interest ("Crop Settings"). When saving the TrackMate file (bottom "Save"), an XML file will be created containing all the processed data.

4. Navigate to the next window to select a detector for segmentation of the specimens (i.e. embryos or larvae). Use the LoG detector for a diameter of the specimen between 5 and 20 pixels. Indicate the estimated specimen size in pixels in the next window ("Estimated Blob Diameter"). To provide an example, a typical annelid larva in the RA treatment experiment is close to 10 pixels in size. "Preview" to inspect the segmentation result. Selected spots are highlighted with a purple circle (see Fig. 2a). Application of a threshold value (here 1.0) will remove many of falsely detected spots (see Fig. 2b). In the following windows, application of different filtering criteria will further lower the number of segmented spots.

5. The first filtering option allows the definition of a quality value, above or below which the spots will be included in the tracking analysis. This value, calculated by the segmentation software, reflects the probability of a spot to represent a real biological specimen. Of note, segmented spots removed in this step will be removed from the metadata, which will speed up the processing time of the calculations. In the current 
analysis, assessing the effects of RA treatments on the swimming behavior of $P$. dumerilii larvae, we used a quality value above 4.4.

6. Select a displayer for the visualization of specimen spots and tracks on the live imaging recordings. For our 2D recordings, we chose the option "HyperStack Displayer". Press "Next".

7. In the next panel, various filtering options are available and can be applied to the data by clicking on the "+" button. Select a filtering feature in the scroll down menu and determine the filtering criteria by manually adjusting the histogram or by pressing "Auto". Included spots are highlighted in the image. For our movies, "Contrast" $(>0.5)$ or "Signal/Noise" $(>1.0)$ were used to ensure unique segmentation of the larvae. This step is critical to lower the number of selected spots before linking them throughout the movie. Importantly, the deselected spot information is saved in the XML file and can be retrieved again by navigating back in the TrackMate window. Press "Next".

8. Select a tracking algorithm to connect the segmented specimens between time points. We chose the "Linear Assignment Problem" (or "LAP") tracker, which includes the feature "Gap-Closing Events" to connect the track of a specimen, even if it disappears in some of the time frames (for example, because the specimen is out of focus or outside the imaging window).

9. To optimize the tracking, apply the following parameters to the tracker: (1) the maximal allowed linking distance of a specimen between two time frames, (2) the maximal distance for gap-closing of a specimen, and (3) the allowed number of frame gaps in the gap-closing feature. The latter two values allow for the connection of a specimen between two separate time points, even if the specimen is undetectable in the period between these two time points. For our movies, we respectively chose 30 pixels, 25 pixels, and a maximum of 2 gap-closing frames. These values were optimal 
for correctly linking the larvae over time, but did leave some larvae untracked. Higher values increasing the allowed specimen distance between time points increased the number of tracked larvae, but also caused the erroneous linking of larvae (i.e. different specimens were connected over time). The number of calculated tracks will be displayed when pressing "Next".

10. Filtering of the tracks is also necessary. Use the "+" button to add filtering parameters from the scroll down menu. Determine the filtering criteria by manually adjusting the histogram or by pressing "Auto". For our purpose, both "Number of Spots in a Track" (>35) and "Track Displacement" (>100) were used for incrementing the ratio of real tracks (see Note 8). The number of remaining tracks is displayed in the TrackMate window as the filtering options are applied to the dataset.

11. In the next window, the visualization of the spots and tracks can be adjusted. We selected "Uniform Color" for the spots and color by "Track Start" for the tracks. Adjust also the "Track Display Mode" to show all tracks or only a selection of the tracks, according to the options in the scroll down menu (see Fig. 3). At this point of the data analysis, an overall impression of swimming path and behavior under different experimental conditions can be displayed (see Fig. 4).

12. In the same window, the tracks can be visualized, inspected, and curated with "TrackScheme". For manual curation, in "Display Tracks", set "Show Local Tracks" to "Backward" and "Limit Frame Depth" to 20, and then open "TrackScheme". When selecting a spot in "TrackScheme", this spot will be highlighted in green in the movie (see Fig. 5). A dragon tail displays the track of the specimen in the last 20 frames of the movie (see Note 9). Use the arrows to move through the movie over time in "TrackScheme" to inspect the path of the specimen. Perform manual curation in "TrackScheme" or the displayer window, for example, to move a spot along the $\mathrm{x}-$ and/or $y$-axis (change the position of the spot with the mouse), to delete a spot (with 
the "D" key), to add a spot (with the "A" key) or to link different tracks (with the "L" key). In our current experiment, we manually curated a minimum of 30 larval tracks per experiment. Save the resulting XML file.

13. Non-corrected tracks can be deleted by selecting them in "TrackScheme" (click the right mouse button and delete with "Remove Spots and Links"). Save the project as XML file under a different name, since the deleted information will be irretrievably lost.

14. Visualize the curated swimming tracks by displaying "Show All Entire Tracks" in the "Display Options" window of TrackMate. Create a picture (or take a screenshot) of the completed swimming tracks to compare the swimming patterns between experimental conditions (see Fig. 6a-f).

15. Visualize the swimming behavior directly by overlaying the specimen tracks on the recorded movie. To do this, set "Show Local Tracks" to "Backward" in the "Display Options" window of TrackMate. Navigate forward two windows and execute the “Capture Overlay” action (see Movie 1) (see Note 10).

16. For calculation of the swimming speed, navigate two windows back to "Display Options". Select "Analysis" to extract all the spot and tracking data. Three files will open: "Track Statistics", "Spots in Tracks Statistics", and "Links in Tracks Statistics". Save these three files in CSV format for further analysis.

17. The larval swimming speed (i.e. the "Track Mean Speed" values) can be extracted directly from the "Track Statistics" file, if the time intervals between time frames in the recordings are identical (see Note 11). However, if the time intervals vary between time frames, as in our case, the speed has to be calculated manually.

18. For manual calculation of the swimming speed, extract the time at each time frame of the movie. This information is available in the metadata of the recordings. Annotate also the time frames covering each of the manually curated specimen tracks ("Frame" 
values from the file "Spots in Tracks Statistics"). Determine the time points of the first and the last time frame of the specimen track to obtain the total duration of the track. Thereafter, extract the corresponding length of the specimen track ("Total Distance Traveled" value from the file "Links in Tracks Statistics"). This value is in pixels and can be converted into a metric value by multiplying the pixel value with the image pixel size $(11.33 \mu \mathrm{m}$, when using the Nikon Digital Sight DS-Fil camera, a 0.6x projection lens and a $0.5 x$ objective lens) (see Note 12). Calculate the swimming speed as total distance traveled divided by total time. In our case, this resulted in values measured in $\mu \mathrm{m} / \mathrm{sec}$.

19. Display the swimming speeds in a box plot for comparison between the different experimental conditions (see Fig. 6g). Free online tools, such as BoxPlotR (http://shiny.chemgrid.org/boxplotr/), can be used for generating the box plots.

20. Perform statistical analyses, like a Student's t-test, to assess the statistical significance of the effects on larval swimming behavior observed with different drugs (see Fig. $6 \mathrm{~g})$. Free statistical analyses can be carried out, for example, by making use of the QuickCalcs program suite provided online by GraphPad Software (https://www.graphpad.com/quickcalcs/).

\section{Notes}

1. To ensure reproducibility of treatment-based experiments, strictly respect handling and care guidelines of the compounds to be used and note that the stability of the drug may be altered once dissolved. Generally avoid repeated freeze-thaw cycles once the compound is in solution. If possible, aliquot stock solutions into small volumes for single use. 
2. It is advisable to assess the optimal treatment concentrations with small trial experiments before initiating the actual live recording experiment. As a rule of thumb, RA treatments in vertebrates are carried out at concentrations between $1 \mathrm{nM}$ and 0.1 $\mu \mathrm{M}$, whereas higher concentrations, between $0.1 \mu \mathrm{M}$ and $5 \mu \mathrm{M}$, are generally used in invertebrates, due to lower ligand binding affinity of most invertebrate RA receptors $(3,12,13)$.

3. ATRA can be ordered in aliquots of $50 \mathrm{mg}$ (Sigma-Aldrich). To obtain a $10 \mathrm{mM}$ ATRA stock solution from $50 \mathrm{mg}$ ATRA, the powder has to be dissolved in $16.66 \mathrm{~mL}$ DMSO (or ethanol). 13cRA can be purchased as aliquots of $100 \mathrm{mg}$ (Sigma-Aldrich), and a $10 \mathrm{mM} \mathrm{13cRA} \mathrm{stock} \mathrm{solution} \mathrm{is} \mathrm{prepared} \mathrm{by} \mathrm{dissolving} \mathrm{the} \mathrm{powder} \mathrm{in} 33.32 \mathrm{~mL}$ DMSO (or ethanol).

4. Temperature influences development. $P$. dumerilii embryos, larvae, and adults are kept at $18^{\circ} \mathrm{C}$ in the laboratory, but can grow at temperatures between $14^{\circ} \mathrm{C}$ and $30^{\circ} \mathrm{C}$ (5). Development generally accelerates at higher temperatures and decelerates at lower temperatures (5). Therefore, in order to guarantee comparability between experiments, it is crucially important to keep all cultures at the same, constant, temperature.

5. Embryos can easily be transferred to individual wells of a 6-well plate, if they are transferred before they start to swim. If the embryos are already swimming, they can be concentrated into a smaller volume of NSW by passing the seawater with the embryos through a $50 \mu \mathrm{m}$ mesh (Fisher Scientific). Since $P$. dumerilii embryos measure between 160 and $200 \mu \mathrm{m}$, they will be retained by the mesh and can be transferred into a small volume of NSW. Keep the mesh in seawater at all times to avoid desiccation of the embryos. To ensure the appropriate dilution of the drug, 0.5 $\mathrm{ml}$ of concentrated embryos should be pipetted into a well containing $2.5 \mathrm{ml}$ of NSW. The final treatment volume of $6 \mathrm{ml}$ will then be reached once the $3 \mathrm{ml}$ drug solution is added (see Subheading 3.1.2). 
6. A detailed guide for the use of TrackMate is available at https://imagej.net/Getting_started_with_TrackMate.

7. Any particle, dirt or shiny part of the 6-well plate can mistakenly be interpreted as a specimen and thus be segmented during the detection process. It is therefore highly recommended to define the movie area in a way that it only contains information related to the specimens to be tracked. Since filtered NSW is used as incubation medium (see Subheading 2.2), the amount of particles and dirt in the culture should be minimal.

8. Note that "Track Displacement" is a measurement of the displacement of a tracked specimen between the first and the last time point and does thus not indicate the length of the track itself. This parameter is useful to spot and remove segmentation and tracking of immobile embryos and larvae.

9. At this step, the consequences of the defined track filtering options will become evident, since some spots will have a dragon tail (i.e. these spots were included and thus tracked), while other spots do not have a dragon tail (i.e. these spots were discarded during the track filtering process).

10. For comparisons of the movies under different experimental conditions, use the "Combine..." option in Fiji (14) to organize the movies next to each other. The number of frames should be the same between movies to use this option. Since the movies are generally recorded for one minute at maximum speed (and thus not at a predefined speed), the number of frames can differ between experimental conditions. If this is the case, individual movies need to be duplicated to obtain the desired frame number by using the "Duplicate..." option in Fiji (14). Use "Label Stacks" to label the experimental condition of each movie and to add a time stamp to the movie. This is possible if a fixed time interval is used. In our case, however, the time intervals vary between time points. We hence wrote a script, which uses the time frame information 
from the supplementary metadata of each movie to label the individual time stamps of each movie in Fiji (14). The experimental condition can be added as well. The script is available from the "TomancakLab" update site in Fiji (14) - click "Manage Update Sites" in the Fiji Updater, then click "Update...". Alternatively, it can be downloaded from https://github.com/xulman/TomancakLab/tree/master/7 putLabels. The web page indicates how to present the time frame information in the supplementary metadata and how to use the script.

11. Make sure that proper time intervals are used. This information can be obtained from the metadata of the movies and should be applied to the image properties and/or the initial TrackMate window.

12. The pixel size of a given image can be determined by dividing the camera pixel size by the magnification of the objective used. In our setup, we used a Nikon Digital Sight DS-Fi1 camera with a camera pixel size of $3.4 \mu \mathrm{m}$, a $0.6 \mathrm{x}$ projection lens, a $0.5 \mathrm{x}$ objective lens, and an optical zoom of 1 . The image pixel size was thus 3.4 $\mu \mathrm{m} /(0.6 * 0.5 * 1)=11.33 \mu \mathrm{m} / \mathrm{pixel}$.

\section{Acknowledgements}

The authors are indebted to Miquel Vila Farré and Jochen Rink for help with microscopy recordings. Mette Handberg-Thorsager is supported by the Deutsche Forschungsgemeinschaft (DFG, grant number TO563/7-1), Vladimir Ulman by the German Federal Ministry of Research and Education (BMBF) under the code 031L0102 (de.NBI) and Detlev Arendt by the European Molecular Biology Laboratory and the European Research Council (BrainEvoDevo no. 294810). 


\section{References}

1. Niederreither K, Dollé P (2008) Retinoic acid in development: towards an integrated view. Nat Rev Genet 9:541-553

2. Cunningham TJ, Duester G (2015) Mechanisms of retinoic acid signalling and its roles in organ and limb development. Nat Rev Mol Cell Biol 16:110-123

3. Handberg-Thorsager M, Gutierrez-Mazariegos J, Arold ST et al (2018) The ancestral retinoic acid receptor was a low-affinity sensor triggering neuronal differentiation. Sci Adv 4:eaao1261

4. Jékely G, Colombelli J, Hausen H et al (2008) Mechanism of phototaxis in marine zooplankton. Nature 456:395-399

5. Fischer AH, Henrich T, Arendt D (2010) The normal development of Platynereis dumerilii (Nereididae, Annelida). Frontiers in Zoology 7:31

6. Randel N, Asadulina A, Bezares-Calderón LA (2014) Neuronal connectome of a sensory-motor circuit for visual navigation. eLife 3:e02730

7. Tosches MA, Bucher D, Vopalensky P et al (2014) Melatonin signaling controls circadian swimming behavior in marine zooplankton. Cell 159:46-57

8. Conzelmann M, Offenburger S, Asadulina A et al (2011) Neuropeptides regulate swimming depth of Platynereis larvae. Proc Natl Acad Sci USA 108:E1174-E1183

9. Conzelmann M, Williams EA, Tunaru S et al (2013) Conserved MIP receptor-ligand pair regulates Platynereis larval settlement. Proc Natl Acad Sci USA 110:8224-8229

10. Zieger E, Schubert M (2017) New insights into the roles of retinoic acid signaling in nervous system development and the establishment of neurotransmitter systems. Int Rev Cell Mol Biol 330:1-84

11. Kane MA, Chen N, Sparks S et al (2005) Quantification of endogenous retinoic acid in limited biological samples by LC/MS/MS. Biochem J 388:363-369 
12. Escriva H, Bertrand S, Germain P et al (2006) Neofunctionalization in vertebrates: the example of retinoic acid receptors. PLoS Genet 2:e102

13. Gutierrez-Mazariegos J, Nadendla EK, Studer RA et al (2016) Evolutionary diversification of retinoic acid receptor ligand-binding pocket structure by molecular tinkering. R Soc Open Sci 3:150484

14. Schindelin J, Arganda-Carreras I, Frise E et al (2012) Fiji: an open-source platform for biological-image analysis. Nat Methods 9:676-682

15. Tinevez JY, Perry N, Schindelin J et al (2017) TrackMate: an open and extensible platform for single-particle tracking. Methods 115:80-90

16. Meijering E, Dzyubachyk O, Smal I (2012). Methods for cell and particle tracking. Methods Enzymol 504:183-200 


\section{Figure captions}

Fig. 1 Launching TrackMate. Defining image properties in the initial TrackMate window.

Fig. 2 Detection of embryos and larvae by TrackMate. (a) Define the diameter of the specimen and inspect by pressing "Preview". Here, many of the segmented spots are larvae (yellow arrow), but TrackMate also detected many other objects (blue arrow). (b) Introducing a threshold can eliminate falsely labeled spots (compare yellow and blue arrows between (a) and (b)).

Fig. 3 TrackMate display options of segmented larvae (i.e. the spots) and tracks. Scroll down menus offer various options for differential visualization. Here, spots were labeled with "Uniform Colors" (pink) and tracks by "Track Start" (indicated by a progressive color change from the beginning to the end of the movie).

Fig. 4 Tracking results, obtained with TrackMate, following treatments of Platynereis dumerilii larvae with retinoic acid (RA). (a) Wild-type (wt) larvae. (b) Control larvae treated with dimethyl sulfoxide (DMSO). (c) Larvae treated with $0.5 \mu \mathrm{M}$ all-trans RA (ATRA). (d) Larvae treated with $1 \mu \mathrm{M}$ all-trans RA (ATRA). (e) Larvae treated with $0.5 \mu \mathrm{M}$ 13-cis RA (13cRA). (f) Larvae treated with $1 \mu \mathrm{M} 13$-cis RA (13cRA). The color of the track indicates the starting time point in the movie, following the color code indicated in (a). Scale bar: 500 $\mu \mathrm{m}$.

Fig. 5 Manual curation of TrackMate larval tracks. To inspect a larval track, select a spot in "TrackScheme" and follow its track over time in the display window. The selected larva is highlighted in green in both "TrackScheme" and display window. Here, the larval track is 
visualized with a dragon tail consisting of the last 20 time frames. Larval tracks can easily be split or linked in "TrackScheme". For splitting, select the linker between two spots, click the right mouse button, and select "Remove Spots and Links". For connecting two tracks, select the end spot of one track and the first spot of the subsequent track and press the "Toogle Linking" button in the "TrackScheme" menu (alternatively, press the "L" key). For deleting multiple spots in a larval track, highlight the spots in "TrackScheme", click the right mouse button, and select "Remove Spots and Links". Spots can be added by pressing the "A" key in the display window. Press "Layout" in the "TrackScheme" menu to update the "TrackScheme" window.

Fig. 6 Analysis of curated larval tracking results following retinoic acid (RA) treatments of Platynereis dumerilii larvae. (a-f) Curated larval tracks using TrackMate. (a) Wild-type (wt) larvae. (b) Control larvae treated with dimethyl sulfoxide (DMSO). (c) Larvae treated with $0.5 \mu \mathrm{M}$ all-trans RA (ATRA). (d) Larvae treated with $1 \mu \mathrm{M}$ all-trans RA (ATRA). (e) Larvae treated with $0.5 \mu \mathrm{M}$ 13-cis RA (13cRA). (f) Larvae treated with $1 \mu \mathrm{M} 13$-cis RA (13cRA). The color of the track indicates the starting time point in the movie, following the color code indicated in (a). Scale bar: $500 \mu \mathrm{m}$. (g) Exogenous RA treatments slow down P. dumerilii larval swimming. Box plots showing the speed of $P$. dumerilii larval swimming following different RA treatments. Data distribution (circles), median values (bold line), and Tukey whiskers are shown. The number of curated larval tracks is indicated (n). An unpaired Student's t-test on the mean value was used for statistical analysis (n.s., non-significant; *, $\mathrm{P}<0.05 ; * *, \mathrm{P}<0.01) . \mathrm{P}=0.9330$ for $\mathrm{DMSO}$ versus wild-type $(w t) ; \mathrm{P}=0.8810$ for DMSO versus $0.5 \mu \mathrm{M}$ ATRA; $\mathrm{P}=0.0001$ for DMSO versus $1 \mu \mathrm{M}$ ATRA; $\mathrm{P}=0.0416$ for DMSO versus $0.5 \mu \mathrm{M} 13 \mathrm{cRA} ; \mathrm{P}=0.0038$ for DMSO versus $1 \mu \mathrm{M}$ 13cRA. 
Movie 1 Curated swimming tracks of wild-type Platynereis dumerilii larvae or P. dumerilii larvae treated with dimethyl sulfoxide (DMSO, control larvae), $0.5 \mu \mathrm{M}$ all-trans retinoic acid (ATRA), $1 \mu \mathrm{M}$ all-trans retinoic acid (ATRA), $0.5 \mu \mathrm{M}$ 13-cis retinoic acid (13cRA) or $1 \mu \mathrm{M}$ 13-cis retinoic acid (13cRA). The trajectories are overlaid on the live imaging recordings. The first 254 time points are shown. The color of the track indicates the starting time point in the movie, following the color code in Figure 6a. Scale bar: $500 \mu \mathrm{m}$. 
Please note that TrackMate is available through Fiji, and is based on a publication. If you use it successfully for your research please be so kind to cite our work:

Tinevez, JY.; Perry, N. \& Schindelin, J. et al. (2017),

TrackMate: An open and extensible platform for

single-particle tracking. Methods 115: 80-90

on PubMed (PMID 27713081)

Target: DMSO_crop

Calibration settings:

$\begin{array}{lll}\text { Pixel width: } & 1.000 & \text { pixel } \\ \text { Pixel height: } & 1.000 & \text { pixel } \\ \text { Voxel depth: } & 1.000 & \text { pixel } \\ \text { Time interval: } & 1.000 & \text { frame }\end{array}$

Crop settings (in pixels, 0 -based):

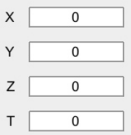

to

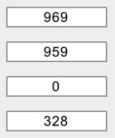

Refresh source

\begin{tabular}{l} 
to \\
to \\
to \\
to 328 \\
\hline
\end{tabular}
source

百 Save 


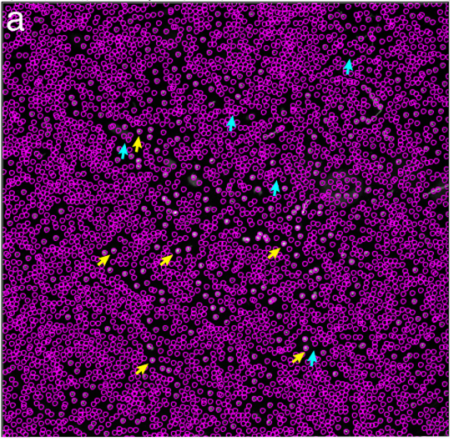

DMSO_crop.tif (50\%)

\section{$1 / 329(0.01$ s); $970 \times 960$ pixels; 8 -bit; $292 \mathrm{MB}$}

\section{b}

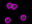

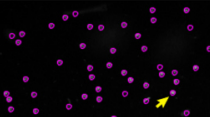

9

Q.

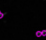

09

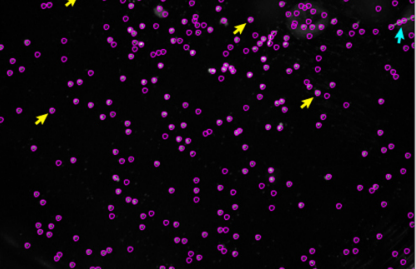

Settings for detector.

\section{LoG detector}

This defector apples a LoG (Laplacian of Gaussian) fiter to the image, wth a sigma sulted to the blob estimated size. Calculations are made in the Fourier space. The maxima in the fatered image are searched for, and maxima too close from eech other are suppressed. A quadratic fitting scheme allows to do sub-pixel localization.

Estmated blob diameter:

Threshold:

\begin{tabular}{|l|}
\hline 10.0 \\
\hline 0.0 \\
\hline
\end{tabular}

$\checkmark$ Use median fitter

$\checkmark$ Do sub-pixel localzation

29 Preview

Found 4568 spots.

Bave

and

Settings for detector.

\section{LoG detector}

This detector applies a LOG (Lapiacian of Gaussian) fiter to the image, with a sigma suited to the blob estimated size. Calculations are mado in the Fourier space. The maxima in the filtered image are searched for, and maxima too close from each other are supprossed. A quadratic fitting scheme allows to do sub-paxel locelization.

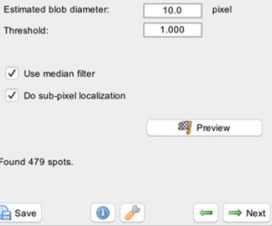




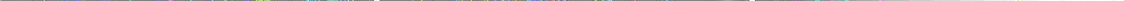




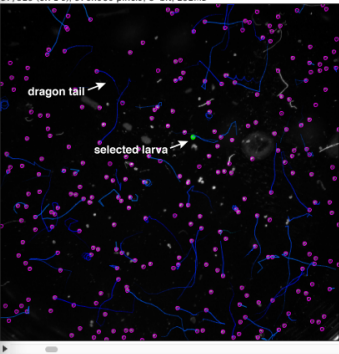




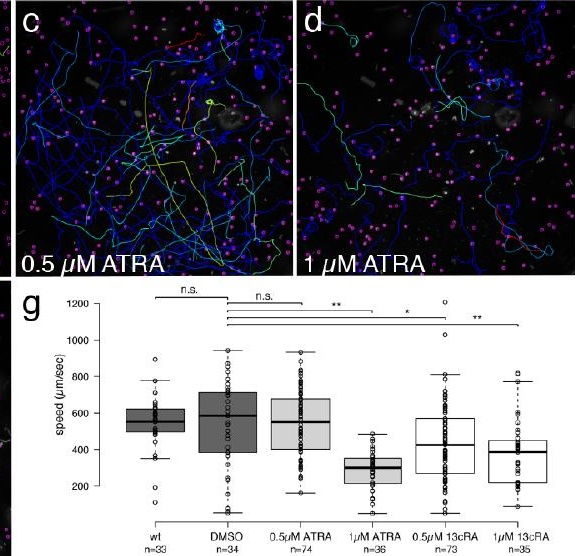

